\title{
Neutropenia: occurrence and management in women with breast cancer receiving chemotherapy
}

\author{
Talita Garcia do Nascimento ${ }^{1}$ \\ Marceila de Andrade ${ }^{2}$ \\ Rosemeire Aparecida de Oliveira ${ }^{3}$ \\ Ana Maria de Almeida ${ }^{4}$ \\ Thais de Oliveira Gozzo ${ }^{5}$
}

Objectives: to identify the prevalence, and describe the management of, neutropenia throughout the chemotherapy treatment among women with breast cancer. Methods: observational study, cycles of chemotherapy. 116 neutropenic events were recorded, and $63.3 \%$ of the patients presented neutropenia at some point of their treatment, $46.5 \%$ of these presenting grade II. The management used was temporary suspension between the cycles and the mean number of delays was 6 days. The study was prospective and longitudinal, where the evaluation of the hematological toxicities was undertaken at each cycle of chemotherapy, whether neoadjuvant or adjuvant. Results: 79 women were included, who received 572 cycles. However, the reasons for the suspensions were the lack of a space in the chemotherapy center, followed by neutropenia. Conclusion: neutropenia is one of the most common and serious adverse events observed during the chemotherapy. Nursing must invest in research regarding this adverse event and in management strategies for organizing the public health system, so as to offer quality care.

Descriptors: Nursing; Breast Neoplasms; Drug Therapy/adverse effects.

\footnotetext{
${ }^{1} \mathrm{MSc}$, Professor, Faculdade Barretos, Barretos, SP, Brazil.

${ }^{2}$ MSc, RN, Prefeitura Municipal de Ribeirão Preto, Ribeirão Preto, SP, Brazil.

${ }^{3}$ Master's student, Escola de Enfermagem de Ribeirão Preto, Universidade de São Paulo, WHO Collaborating Centre for Nursing Research Development, Ribeirão Preto, SP, Brazil.

${ }^{4} \mathrm{PhD}$, Associate Professor, Escola de Enfermagem de Ribeirão Preto, Universidade de São Paulo, WHO Collaborating Centre for Nursing Research Development, Ribeirão Preto, SP, Brazil.

${ }^{5} \mathrm{PhD}$, Professor, Escola de Enfermagem de Ribeirão Preto, Universidade de São Paulo, WHO Collaborating Centre for Nursing Research Development,
} Ribeirão Preto, SP, Brazil.

Corresponding Author:

Thais de Oliveira Gozzo

Universidade de São Paulo. Escola de Enfermagem de Ribeirão Preto

Departamento Materno-Infantil e Saúde Pública

Av. Bandeirantes, 3900

Bairro: Monte Alegre

CEP: 14040-902, Ribeirão Preto, SP, Brasil

E-mail: thaisog@eerp.usp.br
Copyright () 2014 Revista Latino-Americana de Enfermagem This is an Open Access article distributed under the terms of the Creative Commons Attribution Non-Commercial License (CC BY-NC).

This license lets others distribute, remix, tweak, and build upon your work non-commercially, and although their new works must also acknowledge you and be non-commercial, they don't have to license their derivative works on the same terms. 


\section{Introduction}

Neutropenia is characterized by the reduction in white blood cells, with a neutrophil count of below $1.5 \times 10^{9} / \mathrm{L}$. The occurrence of this event varies from $16 \%$ to $81 \%$ among the patients who receive chemotherapy treatment ${ }^{(1-3)}$.

It is one of the principal adverse events $(A E)$ of antineoplastic chemotherapy, and is the one which interferes most in the dosage of the chemotherapy agents. The occurrence of neutropenia is associated with an increase in mortality, morbidity and treatment costs, in addition to the impossibility of predicting which patients will present it ${ }^{(4-5)}$.

The management of this chemotherapy-induced $A E$ is administered in different ways in clinical practice. In general, the strategies are limited to the reduction of the dose of the chemotherapy agents, postponement between the cycles, and the use of colony-stimulating factors, although these are considered common clinical practices during the treatment of patients with primary breast cancer who present AEs, such as episodes of neutropenia( ${ }^{(6)}$.

In spite of these strategies being widely used, clinical studies have shown that these modifications in the dosage or in the time scheduled can have as a consequence an inferior result in the final quality of the therapy ${ }^{(7)}$.

The efficacy of the assistance and the treatment given to the patient are fundamental for the quality of the health care. The occurrence of neutropenia indicates the need for nursing to define protocols for evaluation and follow-up, which includes the AEs, the degrees of compromise, and their management ${ }^{(1)}$.

The standards of neutropenia management vary from one health center to another. The development of nursing care protocols for the management of chemotherapy-induced neutropenia may allow more patients to benefit from: treatment with the complete dose undertaken within the schedule; reduction in variety of conduct in the assistance provided; promotion of the efficacious use of colony-stimulating factor; help in taking decisions regarding contradictory situations; and, principally, from improvement in the quality of the care for the patient, which leads to improvement in the results of the treatment ${ }^{(8)}$.

In the Gynecologic Oncology and Mastology outpatient clinic of the Hospital das Clínicas, University of São Paulo at Ribeirão Preto, Medical Faculty, of the (HCFMRP-USP), there are no nursing care protocols for attending women with gynecological or breast cancer during their treatment, which makes it difficult to standardize the guidance and information provided to the woman and her caregivers by the nursing team. In the women's accounts, one can observe divergent or very often contradictory information. This hinders decision-making regarding the AEs of the chemotherapy treatment and the building of trust in the team. One may also note the absence of Brazilian studies directed at the management of neutropenia in the area of Nursing, as well as the implementation of specific protocols which help in improving the quality of the care given.

In the light of the above, this study's objective was to identify the prevalence of, and describe the management of, neutropenia throughout the chemotherapy treatment among women with breast cancer.

\section{Method}

This study is part of the project titled "Evaluation of the quality of life, anxiety, depression and toxicity to the chemotherapy treatment in women with breast cancer", approved by the HCFMRP-USP's Ethics Committee, under Process HCRP 1460/2009.

Focussing on chemotherapy-induced neutropenia, an observational, longitudinal and prospective study was undertaken, evaluating this $A E$ at each cycle of chemotherapy whether neodjuvant or adjuvant, in women with breast cancer under treatment at the Mastology Outpatient Center of the Teaching Hospital of the Ribeirão Preto Faculty of Medicine of the University of São Paulo (HCFMRP-USP).

Based on a calculation of 100 new cases/year of breast cancer attended in the above-mentioned outpatient center, the study recruited all the women who started chemotherapy treatment in the period April 2009 - March 2010, with the inclusion criteria: women aged over 19 years old with a diagnosis of breast cancer; who were beginning chemotherapy treatment for the first time, neoadjuvant or adjuvant. The following were considered exclusion criteria: women who could not express themselves without the help of another person, who presented metastatic disease at diagnosis or during the treatment, who presented another malignant tumor at diagnosis, who had previously been treated for breast cancer or for another type of malignant tumor, and women in the puerperal stage of pregnancy.

The study included 83 women with breast cancer, who had started neoadjuvant or adjuvant chemotherapy treatment. There were no refusals to participate; 
however, during the follow up, two women abandoned the chemotherapy treatment due to intolerance of the AEs, one requested to be excluded from the research, and one was diagnosed with metastasis. As a result, the sample was made up of 79 women.

For data collection, an instrument was used with information on identification, data on the diagnosis, the result of the complete blood counts ( $\mathrm{CBCs}$ ) undertaken prior to each cycle of chemotherapy, the dosage of the chemotherapy agents used in the neoadjuvant and adjuvant settings, data of possible episodes of hospitalization during the chemotherapy treatment and, also, data on the surgical procedure. This information was collected at each cycle in interviews with the women participating, as was complementary information such as the results of tests, obtained from their hospital records. Neutropenia and the socio-demographic variables of age, race, co-morbidity and the staging of the cancer were defined as the study's variables.

Based on the study's objectives, descriptive analysis (frequency, mean, median and standard deviation) of all the variables was undertaken, as was the descriptive analysis (frequency, mean, median and standard deviation) of the variable neutrophil, for each cycle. IBM, Armonk, NY, U.S.A.) was used for this.

\section{Results}

Among the 79 women who participated, the age varied from 29 to 69 years old, with $34.2 \%$ falling into the age range of 51 to 60 years old, and $24.1 \%$ being aged below 40 years old. The participants' mean age was 48.4 years old, with a standard deviation of 9.67 years (Table 1 ).

Considering the clinical stages of breast cancer presented, the most observed was IIa in $25.3 \%$ of the women, followed by IIb and IIIb with a frequency of $21.5 \%$ for each one (Table 1 ).

In relation to the therapy used, $50.6 \%$ of the women received neoadjuvant chemotherapy treatment, while $49.4 \%$ received adjuvant chemotherapy treatment. The chemotherapy protocols used, both for the neoadjuvant treatment and the adjuvant, were FEC (Fluorouracil, Epirubicin and Cyclophosphamide), EC-T (Epirubicin, Cyclophosphamide and Paclitaxel), EC-TH (Epirubicin, Cyclophosphamide, Paclitaxel and Trastuzumab) and EC (Epirubicin and Cyclophosphamide). The choice of scheme is made in line with the institution's protocol, which stipulates as criteria the definition of the clinical staging and the histological diagnosis, as well as the immunohistochemical panel for hormone receptors and HER2/neu.

Table 1 - Distribution of women who received chemotherapy for breast cancer, by age, staging of the disease and nutritional status. Ribeirão Preto, State of São Paulo (SP), Brazil, $2010(\mathrm{~N}=79)$

\begin{tabular}{lc}
\multicolumn{1}{c}{ Characteristic } & $\begin{array}{c}\text { Number and percentage } \\
(\%) \text { of women }\end{array}$ \\
\hline Age & $1(1.3 \%)$ \\
Below 30 years old & $18(22.8 \%)$ \\
31 to 40 years old & $25(31.6 \%)$ \\
41 to 50 years old & $27(34.2 \%)$ \\
51 to 60 years old & $8(10.1 \%)$ \\
61 to 69 years old & \\
Staging of the disease & $2(2.5 \%)$ \\
In situ & $5(6.3 \%)$ \\
la & $20(25.3 \%)$ \\
Ila & $17(21.5 \%)$ \\
Ilb & $15(19 \%)$ \\
IIla & $17(21.5 \%)$ \\
IIlb & $3(3.8 \%)$ \\
No record & \\
Body composition & $17(1 \%)$ \\
Below weight $\left(<18.5 \mathrm{Kg} / \mathrm{m}^{2}\right)$ & $31(41 \%)$ \\
Normal weight $\left(18.5-24.99 \mathrm{Kg} / \mathrm{m}^{2}\right)$ & $27(36 \%)$ \\
Overweight $\left(\geq 25 \mathrm{Kg} / \mathrm{m}^{2}\right)$ & \\
Obesity $\left(\geq 30 \mathrm{Kg} / \mathrm{m}^{2}\right)$ & $17 \%)$ \\
\hline
\end{tabular}

A total of 572 chemotherapy cycles was undertaken, with 301 cycles for neoadjuvant treatment and 271 for adjuvant treatment. During the undertaking of the 572 chemotherapy cycles, various complications were identified, which directly influenced the duration of the treatment. Of the 79 women studied, 30.4\% were hospitalized during the treatment, totalling 30 hospitalizations, excluding the hospitalizations caused by undertaking complementary tests or surgical procedures. It stands out that $33.33 \%$ (10 hospitalizations) were due to febrile neutropenia, eight to dehydration, two to urinary tract infections, two to pneumonia, and eight were for other reasons.

According to the hematological tests collected prior to each cycle of chemotherapy, of the 79 women who participated in the study, $63.3 \%$ presented neutropenia at some point during the chemotherapy treatment. Of these, $16.5 \%$ developed the event just once, $20.2 \%$ presented neutropenia twice during the chemotherapy, $16.5 \%$ three times, and $10.1 \%$ had four neutropenic episodes.

The occurrence of neutropenia was observed both in neoadjuvant treatment and in adjuvant treatment. 
Of the 40 women who received the neoadjuvant treatment, $24(60 \%)$ developed neutropenia at some point of the chemotherapy, which represented $48 \%$ of all the neutropenic episodes verified in the study. In the adjuvant treatment, greater occurrence of neutropenia was observed, as, among the 39 participants, it was presented by $26(66.7 \%)$, representing $52 \%$ of the 50 neutropenic events.

According to the chemotherapy protocol, 31 women used EC-T/EC-TH, of whom $67.7 \%$ developed neutropenia. Among the 48 patients treated with FEC/ $E C$, on the other hand, $60.4 \%$ presented neutropenia.

A total of 116 neutropenic episodes was observed during the study. Of these, $9.5 \%$ were classified as grade I, $46.5 \%$ grade II, $31.9 \%$ grade III and $12.1 \%$ as grade IV. The hematological results obtained prior to initiating the third cycle of chemotherapy showed a greater occurrence of neutropenic events $(23.3 \%)$ in this stage of the treatment (Table 2).

The highest number of occurrences of grades III and IV neutropenia was observed in the tests made prior to the third cycles (32.4\%) and the highest number of occurrences of neutropenia grade IV, in the tests which preceded the second cycles $(42.8 \%)$, indicating the need for continuous evaluation of symptoms, and vigilance during this phase of the treatment.

The mean age of the women who presented neutropenia grade III at some point of their treatment was 49.63 years old, while that of those who presented grade IV was 43.2 years old. The BMI of the patients who presented grade III and grade IV of neutropenia was $28.3 \mathrm{Kg} / \mathrm{m}^{2}$ and $28.5 \mathrm{Kg} / \mathrm{m}^{2}$, respectively.

Table 2 - Neutropenic episodes observed in women with breast cancer who received chemotherapy treatment, by the grade of neutropenia and cycle of chemotherapy. Ribeirão Preto, SP, Brazil, 2010

\begin{tabular}{|c|c|c|c|c|c|c|c|c|c|}
\hline & Cycle 1 & Cycle 2 & Cycle 3 & Cycle 4 & Cycle 5 & Cycle 6 & Cycle7 & Cycle 8 & Total (\%) \\
\hline Grade I & 0 & 2 & 1 & 2 & 2 & 2 & 2 & 0 & $11(9.5 \%)$ \\
\hline Grade II & 0 & 6 & 12 & 13 & 15 & 6 & 0 & 2 & $54(46.5 \%)$ \\
\hline Grade III & 0 & 7 & 12 & 8 & 8 & 2 & 0 & 0 & $37(31.9 \%)$ \\
\hline Grade IV & 0 & 6 & 2 & 1 & 1 & 4 & 0 & 0 & $14(12.1 \%)$ \\
\hline Total & 0 & 21 & 27 & 24 & 26 & 14 & 2 & 2 & 116 (100\%) \\
\hline
\end{tabular}

Neutropenia was responsible for the temporary suspension of the treatment in 42 cycles of the 159 undertaken by women who used the EC-T/ EC-TH protocols, and of the 118 cycles of FEC/EC, 44 were not administered in the space of time stipulated by the protocol, for the same reason.

Delays occurred between the cycles of chemotherapy, the mean being 6.3 days. It stands out that the cycles' delays did not occur solely due to the management of neutropenia, being also related to the management of the health system, with a lack of spaces in the chemotherapy center. In spite of this difference - between the management of the neutropenia and the management of the health system - the authors present the reasons for the delays grouped in line with the chemotherapy protocol received by the participant.

According to the data presented in Tables 3 and 4, the lack of spaces in the Chemotherapy Center took first place, and neutropenia the second, among the reasons for delays between the chemotherapy cycles.

Another possibility for managing neutropenia, reduction in the dose, was observed in this study only for one participant. Other possible means of management, such as the use of colony-stimulating factors, were not used.

Table 3 - Distribution of the women who received chemotherapy for breast cancer, by reason for the delay between the cycles. Ribeirão Preto, SP, Brazil, $2010(\mathrm{~N}=48)$

\begin{tabular}{|c|c|c|c|c|c|c|c|c|}
\hline $\begin{array}{l}\text { Reason for delay for the } \\
\text { EC-T/EC-TH Protocol }\end{array}$ & $\begin{array}{l}\text { Cycle } 2 \\
(n=48)\end{array}$ & $\begin{array}{c}\text { Cycle } 3 \\
(n=48)\end{array}$ & $\begin{array}{l}\text { Cycle } 4 \\
(n=48)\end{array}$ & $\begin{array}{c}\text { Cycle } 5 \\
(n=48)\end{array}$ & $\begin{array}{l}\text { Cycle } 6 \\
(n=47)\end{array}$ & $\begin{array}{l}\text { Cycle } 7 \\
(n=46)\end{array}$ & $\begin{array}{l}\text { Cycle } 8 \\
(n=42)\end{array}$ & Total \\
\hline Lack of a space & 8 & 11 & 11 & 7 & 8 & 3 & 1 & 49 \\
\hline Neutropenia & 7 & 10 & 10 & 12 & 1 & 0 & 2 & 42 \\
\hline Infection & 1 & 1 & 1 & 1 & 0 & 0 & 1 & 5 \\
\hline Others & 6 & 7 & 8 & 7 & 11 & 18 & 6 & 63 \\
\hline Total & 22 & 29 & 30 & 27 & 20 & 21 & 10 & 159 \\
\hline
\end{tabular}


Table 4 - Distribution of the women who received chemotherapy for breast cancer, by reason for the delay between the cycles. Ribeirão Preto, SP, Brazil, $2010(\mathrm{~N}=31)$

\begin{tabular}{|c|c|c|c|c|c|c|}
\hline Reason for the delay for the FEC/EC Protocol & $\begin{array}{c}\text { Cycle } 2 \\
(n=31)\end{array}$ & $\begin{array}{c}\text { Cycle } 3 \\
(n=31)\end{array}$ & $\begin{array}{c}\text { Cycle } 4 \\
(n=31)\end{array}$ & $\begin{array}{l}\text { Cycle } 5 \\
(n=31)\end{array}$ & $\begin{array}{c}\text { Cycle } 6 \\
(n=31)\end{array}$ & $\begin{array}{c}\text { Total } \\
\text { Number }\end{array}$ \\
\hline Neutropenia & 7 & 10 & 10 & 8 & 9 & 44 \\
\hline Lack of a space & 8 & 11 & 11 & 2 & 1 & 33 \\
\hline Infection & 1 & 1 & 1 & 1 & 0 & 4 \\
\hline Others & 6 & 7 & 8 & 7 & 9 & 37 \\
\hline Total & 22 & 29 & 30 & 18 & 19 & 118 \\
\hline
\end{tabular}

\section{Discussion}

The development of myelosuppression during chemotherapy is influenced as much by the characteristics of the drugs used as by the conditions presented by the patient. Therefore, the association of myelotoxicity with the activity of the treatment is not always $\operatorname{direct}^{(9)}$.

Although the risk factors for neutropenia during chemotherapy treatment are not well defined, current studies point to: advanced age ( $\geq 65$ years), female sex, low performance status and nutritional status, reduced body surface area, reduction in blood cell count prior to starting treatment (reduced number of lymphocytes and of hemoglobin) and previous involvement of the bone marrow $^{(4,10)}$.

The mean age of patients who participate in randomized clinical trials varies from 46 to 49 years old and is similar to that found in this study. However, it was possible to observe that the percentage of young women, from 31 to 40 years old, with mammary neoplasms has increased considerably, representing $22.8 \%$ in the sample studied. However, it stands out that in current studies the authors present the mean age of all the participants, without distinguishing between the age ranges which present neutropenia during the treatment or $\operatorname{not}^{(2,11-12)}$.

Regarding the choice, use and activity of the drugs, current data indicates that both for adjuvant and neoadjuvant treatment of breast cancer, chemotherapy regimens based on anthracyclines and taxanes are considered first choice. The introduction of the taxanes and the increase in the doses of the anthracyclines favor greater occurrence of myelotoxicities, resulting in the risk of febrile neutropenia and infections during the treatment $^{(13)}$.

Neutropenia is the event which most influences the limit of the dosage of the chemotherapy agent and the scheduling of the cycles during the treatment. Patients who present chemotherapy-induced neutropenia have a risk of potentially fatal infections. Although the reduction of the dose of chemotherapy or the delay of the subsequent cycle of treatment and, consequently, the reduction of the relative dose intensity, can limit the myelotoxicity, these actions can also impact negatively on the result of the treatment, and must be avoided(14).

These delays or reductions of dose minimize the toxic sequelae of the treatment in patients whose blood count has not recovered appropriately. Such practices can have a significant impact on the survival results, in the same way that they reduce the intensity of the dose received. However, in order to obtain the maximum benefit from the chemotherapy treatment, the maintenance of the total dose calculated must be maintained as an important response factor to the treatment, as for every seven days of delay in the chemotherapy treatment, there is a reduction of approximately $5 \%$ of the intensity of the dose ${ }^{(6)}$.

A limited number of studies has shown that the absence of myelotoxicity during the chemotherapy is associated with a worse prognosis, which suggests that myelodepression may represent an important biological marker for the effect of the treatment. In addition to this, other researchers have emphasized the potential importance of adapting the chemotherapy dose based on the hematological toxicity, as the absence of this may be the result of underdosage. However, prospective studies are necessary to evaluate whether the dosage of the drug, guided by the occurrence of toxic effects, can improve the efficacy of standard regimes ${ }^{(10,15)}$.

Furthermore, the direct effects caused during the treatment, and the chemotherapy-induced neutropenia, have a detrimental effect on the patients' quality of life. In clinical practice, so as to reduce the risk of infection, the neutropenic patients are instructed to follow strict advice, to avoid crowds, not to eat raw fruit/ vegetables and to monitor their temperature regularly. Running a fever with neutropenia is considered a medical emergency, and those who present this event generally need hospitalization and treatment with intravenous broad-spectrum antibiotics. This being so, the hospital 
environment, the separation of members of the family and the undertaking of invasive procedures, and the fear of infection becoming established or of failures occurring in the treatment, as well as the feeling of being close to death, contribute to a substantial reduction in patients' well-being ${ }^{(16-17)}$.

The present study considered the general management of neutropenia, irrespective of the health professional who provided it. While emphasizing that it was not possible to identify the work undertaken by the nursing team among the actions undertaken, these remaining centered on the medical team, the authors believe that preventing the risk of neutropenic complications involves a collaborative approach, with the active and direct participation of nurses, the patient, and family members. In care directed at the neutropenic patient, Nursing is fundamental in the rapid and efficacious identification of neutropenia and the other events which can occur, and in ensuring the continuity of the care provided. In this way, it falls to the nurse to advise the patients and their family members on the consequences of neutropenia before chemotherapy is started, warning them about the importance of adhering to the planned dose and treatment schedule, the need to attend consultations, laboratory monitoring, and the administration of growth factor, if necessary ${ }^{(9)}$.

Oncology nursing, in addition to providing safety to the patients throughout the antineoplastic treatment process, aims to increase their life expectancy with quality and not only to cure; rather, it aims to assist the human being as a whole. Therefore, the assistance provide by Nursing in the routine of the care must be grounded in the quality work directed towards the teaching of self-care, with the objective of protecting the autonomy and the improvement of the patients' quality of life, and must allow the recognition and the valuing of the professional through the establishment of a positive and empathetic relationship between the person who cares and the person who is cared for ${ }^{(18)}$.

The need to establish efficient comprehensive care for the oncological patient requires great mobilization of the entire health system, from the training of the professionals who shall provide the direct care to the infrastructure of the service that the patient shall use.

In a study which evaluated the management of the Nursing service in Brazilian public hospital institutions, the nursing team reports satisfaction regarding the care given to the client, in relation to the qualification of the work, the dedication and the humanization in the attendance, and the communication. On the other hand, they feel uncomfortable because of the service, which lacks adequate facilities and sufficient human resources to care, as well as material in adequate quantities, and appropriate furniture(19).

In research undertaken in 2008 in the same institution as the present study, which evaluated 72 women with breast cancer in neoadjuvant or adjuvant treatment, it was observed that of the 558 chemotherapy cycles undertaken, there were 152 delays between the cycles during the treatment. It is worth emphasizing that 82 of these delays were caused because of alterations detected in the hematological tests, while only six were caused by lack of a space in the chemotherapy center(20).

Considering the data from the present study, one can observe that the number of delays between the cycles of chemotherapy resulting from lack of a space increased, as, of the 277 delays recorded between the cycles of chemotherapy, 82 occurred as a result of the lack of a space in the chemotherapy center. It is asked whether the service is aware of the number of delays, as that presented in this article refers only to the cases of breast cancer, and whether the service is prepared to receive the increase in demand from the cases of cancer.

Even with all the advances undertaken in Brazilian public health policy, with emphasis on comprehensive care for the oncological patients, little has been studied regarding the managing of the health services which attend this type of patient. This hinders the analysis of the suitability of the time periods for undertaking the treatment, such as chemotherapy. In the Brazilian Technical Bases Manual, it is specified that adjuvant chemotherapy should be initiated, at the most, between 30 and 60 days post-surgery ${ }^{(21)}$. However, the time to be waited for undertaking the other chemotherapy cycles is not specified.

The current conditions of saturation in the public health services contribute to privation of citizenship, both of the service users, who receive health care which lacks quality, and of the health workers, who exercise their professional activities under inadequate working conditions. This process makes both parties vulnerable, although in different ways ${ }^{(22)}$.

The health organizations, among these the hospitals, as well as the various types of health professional, have invested in the search for new management strategies which may make it possible to conciliate the reduction of costs, the improvement of the quality of the services offered, and - principally - the clients' satisfaction. 


\section{Conclusion}

The occurrence of neutropenia, observed in the present study, is in accordance with the results of international research. As this is characterized as one of the more common and serious AEs which can occur during antineoplastic chemotherapy, it is an important question to be investigated throughout the treatment.

For decades, Nursing has classified this event as one of the principal axes for carrying out research, in search of results which may indicate improvement in the care given to neutropenic patients. Based on this information, it becomes possible for oncological nurses to perform a key role in improving the care directed at this patient group.

It is through investing in new management strategies able to achieve improvements in the work conditions of health professionals and in the organization of the public health system that the principal objective of the care for oncological patients shall be achieved: the offering of quality care, protecting their autonomy, recognizing and valuing the professional who assists them, and establishing an empathetic relationship between both.

\section{References}

1. Gozzo TO, Nascimento TG, Panobianco MS, Almeida AM. Ocorrência de neutropenia em mulheres com câncer de mama durante tratamento quimioterápico. Acta Paul Enferm. 2011;24(6):810-4.

2. Ramaswamy B, Povoski SP, Rhoades C, Allen J, Hauger $M$, Young $D$, et al. Phase II trial of neoadjuvant chemotherapy with docetaxel followed by epirubicin in stage II/III breast cancer. Breast Cancer Res Treat.2005;93(1):67-74

3. Hirano A, Shimizu T, Imamura $H$, Watanabe $O$, Kinoshita J, Okabe T, et al. The combination of epirubicin plus docetaxel as neoadjuvant chemotherapy in locally advanced breast cancer. Anticancer Res. 2006; 26(1B): 581-4.

4. Lyman $\mathrm{GH}$, Lyman $\mathrm{CH}$, Agboola O. Risk models for predicting chemotherapy-induced neutropenia. Oncologist. 2005;10(6):427-37.

5. Schwenkglenks $M$, Jackisch $C$, Constenla $M$, Kerger JN, Paridaens R, Auerbach $L$, et al. Neutropenic event risk and impaired chemotherapy delivery in six European audits of breast cancer treatment. Support Care Cancer. 2006;14(9):901-9.

6. Kim WY, Woo SU, Seo JH, Son GS, Lee JB, Bae JW. Toxicities, dose reduction and delay of docetaxel and paclitaxel chemotherapy in breast cancer without distant metastases. J Cancer Res Ther. 2011;7(4):412-5.

7. Leonard RC, Miles D, Thomas R, Nussey F; UK Breast Cancer Neutropenia Audit Group. Impact of neutropenia on delivering planned adjuvant chemotherapy: UK audit of primary breast cancer patients. $\mathrm{Br}$ J Cancer. 2003;89(11):2062-8.

8. Dolan S, Crombez $\mathrm{P}$, Munoz M. Neutropenia management with granulocyte colony-stimulating factors: from guidelines to nursing practice protocols. Eur J Oncol Nurs. 2005;9 Suppl 1:S14-23.

9. Koutras AK, Kalofonos HP Myelotoxicity in cancer patients treated with chemotherapy: negative or positive prognostic factor? Clin Cancer Res. 2008;14(22):7579.

10. Lyman GH, Dale DC, Crawford J. Incidence and predictors of low dose-intensity in adjuvant breast cancer chemotherapy: a nationwide study of community practices. J Clin Oncol. 2003;15;21(24):4524-31.

11. Espinosa E, Morales S, Borrega P, Casas A, Madroñal

C, Machengs I, et al. Docetaxel and high-dose epirubicin as neoadjuvant chemotherapy in locally advanced breast cancer. Cancer Chemother Pharmacol. 2004; 54(6): 546-52.

12. Amat S, Bougnoux $P$, Penault-Llorca F, Fétissof $F$, Curé $\mathrm{H}$, Kwiatkowski $\mathrm{F}$, et al. Neoadjuvant docetaxel for operable breast cancer induces a high pathological response and breast-conservation rate. $\mathrm{Br}$ J Cancer. 2003;88(9):1339-45.

13. Debled M, Houédé N, Madranges N, Donamaria C, Floquet A, Durand M, Mauriac L. Does chemotherapyinduced neutropaenia result in a postponement of adjuvant or neoadjuvant regimens in breast cancer patients? Results of a retrospective analysis. $\mathrm{Br}$ J Cancer. 2007;97(12):1642-7.

14. Schwenkglenks $M$, Pettengell $R$, Jackisch $C$, Paridaens R, Constenla M, Bosly A, et al. Risk factors for chemotherapy-induced neutropenia occurrence in breast cancer patients: data from the INC-EU Prospective Observational European Neutropenia Study. Support Care Cancer. 2011;19(4):483-90.

15. Koutras AK, Fountzilas G, Dafni U, Dimopoulos MA, Pectasides D, Klouvas G, et al. Myelotoxicity as a prognostic factor in patients with advanced breast cancer treated with chemotherapy: a pooled analysis of two randomised trials conducted by the Hellenic Cooperative Oncology Group. Anticancer Res. 2008; 28(5B):2913-20.

16. Lyman $\mathrm{GH}$, Kuderer NM Filgrastim in patients with neutropenia: potential effects on quality of life. Drugs. 2002;62 Suppl 1:65-78. 
17. Padilla G, Ropka ME. Quality of life and chemotherapyinduced neutropenia. Cancer Nurs. 2005;28(3):167-71. 18. Menezes MFB, Camargo TC, Guedes MTS, Alcantra LFFL. Cancer, poverty and human development: challenges for nursing care in oncology. Rev. Latino-Am. Enfermagem. 2007;15(spe):780-5.

19. Barreto SS, Pereira MN, Santos JA, Neves EP. (Des) conforto de trabalhadores de enfermagem: uma questão de (in)justiça social. Rev Bras Enferm. 2003;56(6):615-8.

20. Gozzo TO. Toxicidade ao tratamento quimioterápico em mulheres com câncer de mama [tese]. Ribeirão Preto: Escola de Enfermagem da Universidade de São Paulo; 2008.

21. Ministério da Saúde (BR), Secretaria de Atenção à Saúde, Departamento de Regulação, Avaliação e Controle. [Internet]. Manual de bases técnicas da oncologia - SIA/ SUS - sistema de informações ambulatoriais. 2010 [acesso 12 jan 2013]; Disponível em: http://portal. saude.gov.br/portal/arquivos/pdf/manual3_drac.pdf

22. Bellato R, Pereira WR. O gerenciamento em enfermagem frente à pauperização das condições materiais de trabalho. Rev Bras Enferm. 2004;57(4):479-8. 